\title{
Neurobiology of Arousal and Sleep: Updates and Insights Into Neurological Disorders
}

\author{
Miranda M. Lim ${ }^{1,2,3}$ • Ronald Szymusiak ${ }^{4,5,6}$
}

Published online: 30 April 2015

(C) Springer International Publishing AG (outside the USA) 2015

\begin{abstract}
Brain activation during wakefulness is sustained by multiple arousal systems. Dysfunction of one or more arousal systems is a feature of neurological disorders associated with hypersomnolence and/or sleep-wake cycle disturbance. Narcolepsy, Alzheimer's disease (AD), Parkinson's disease (PD), and traumatic brain injury appear to involve hypocretin (HCT) and possibly histamine insufficiency as a mechanism related to excessive daytime sleepiness. Loss of cholinergic neurons in $\mathrm{AD}$ and of dopamine neurons in PD contributes to sleepwake disturbance. GABAergic neurons in the preoptic hypothalamus and rostral medulla promote sleep through inhibition of arousal systems. Pathology of preoptic sleep regulatory circuits is correlated with sleep disturbance in AD. An unidentified endogenous somnogen that potentiates the actions of gamma-aminobutyric acid (GABA) is present in the cerebrospinal fluid (CSF) of patients with primary hypersomnia.
\end{abstract}

This article is part of the Topical Collection on Sleep and Neurological Disorders

Ronald Szymusiak

rszym@ucla.edu

1 Sleep Disorders Laboratory, Division of Hospital and Specialty Medicine, VA Portland Health Care System, Portland, OR, USA

2 Department of Medicine, Oregon Health \& Science University, Portland, OR, USA

3 Department of Neurology and Behavioral Neuroscience, Oregon Health \& Science University, Portland, OR, USA

4 Research Service (151A3), VA Greater Los Angles Healthcare System, 16111 Plummer Street, Los Angeles, CA 91343, USA

5 Department of Medicine, David Geffen School of Medicine, University of California, Los Angeles, CA, USA

6 Department and Neurobiology, David Geffen School of Medicine, University of California, Los Angeles, CA, USA
Descending pathways from the dorsal lateral pons to the ventral medulla and spinal cord are responsible for the inhibition of spinal motoneurons during rapid eye movement (REM) sleep and are implicated in human REM sleep behavior disorder.

Keywords Monoamines $\cdot$ Hypocretin $\cdot$ Orexin ·

Acetylcholine · Dopamine · Glutamate · Galanin · Preoptic hypothalamus · Basal forebrain - Tuberomammillary nucleus . Locus coeruleus $\cdot$ Laterodorsal tegmentum · Alzheimer's disease $\cdot$ Parkinson's disease $\cdot$ Traumatic brain injury

\section{Introduction}

As in all areas of modern neuroscience, rapid progress is being made in understanding the cellular and molecular mechanisms and organization of brain circuits that regulate sleep and wakefulness. It is clear that sleep-wake regulatory mechanisms identified in rodents are highly conserved and that fundamental neurobiological investigations in animal models can inform our understanding sleep disturbances in human disorders. Here we briefly review the current status of knowledge about the functional neuroanatomy and neuropharmacology of sleep and arousal regulation, and attempt to integrate this knowledge with the known neuropathology in some common neurological disorders associated with sleep-wake abnormalities.

\section{Arousal Mechanisms}

Generalized electrographic, behavioral, and autonomic activation during waking emerges from the activity of neurochemically specified arousal systems located in the brain 
stem, posterior and lateral hypothalamus, and basal forebrain. These include neurons that express histamine (HA), serotonin (5HT), noradrenalin (NA), acetylcholine $(\mathrm{ACH})$, glutamate (GLU), dopamine (DA), or hypocretin/orexin (HCT). Each of these neurotransmitter/neuropeptide systems participates in specific aspects of cognition, behavior, sensory processing, and/or autonomic control during waking, but here we focus on their collective contribution to general arousal (Table 1).

Arousal systems can impact neocortical activity indirectly through projections to the thalamus, lateral hypothalamus, or basal forebrain, while others send direct projections to the cortex. There are differences in the pattern and intensity of neuronal activity across sleep-waking states among the arousal systems which has implications for potential roles in sleepwake disturbance associated with neurological disorders.

Monoaminergic Neurons HA neurons in the hypothalamic tuberomammillary nucleus (TMN), 5HT neurons in the dorsal raphe nucleus (DRN), and NA neurons in the locus coeruleus (LC) are characterized by aggregated cell bodies in distinct nuclei with projections to widespread forebrain and brain stem targets [1-3]. All three nuclei target the thalamus, hypothalamus, and basal forebrain and send direct projections to the cortex. Descending projections target visceromotor and somatic motor cranial nerve nuclei.

Monoaminergic neurons also share a "REM off" discharge pattern, characterized by tonic discharge rates during waking, reduced discharge during non-REM sleep, and cessation of activity during REM sleep $[4,5]$. Drugs that promote release or inhibit reuptake of one or more monoaminergic systems promote waking. Drugs that antagonize the postsynaptic actions of the monoamines can be sedating [5].

Acetylcholine ACH-containing neurons are localized in the dorsolateral [6] pontomesencephalic reticular formation, including the pedunculopontine tegmental (PPT) and laterodorsal tegmental (LDT) nuclei and in the basal forebrain
[6]. ACH neurons in the LDT/PPT project to the thalamus and hypothalamus, and those in the basal forebrain project to the limbic system and neocortex. Neurons in both groups exhibit higher rates of discharge in both waking and REM than in NREM sleep $[6,7]$. ACH neurons in the BF contribute to the generation of gamma oscillations in neocortical circuits during activated behavioral states [6]. In mice expressing the light-sensitive excitatory opsin channelrhodopsin-2 in basal forebrain $\mathrm{ACH}$ neurons, light-induced activation during NREM sleep evokes short-latency EEG and behavioral arousal [8].

Dopamine DA-containing neurons implicated in arousal are primarily localized in the substantia nigra and the adjacent ventral tegmental area of the midbrain [9]. At the level of the basal ganglia, effects of DA on sleep and arousal are complex, with DA receptors in the nucleus accumbens associated with arousal and DA signaling in the external segment of the globus pallidus implicated in sleep induction $[9,10]$. DAcontaining wake-active neurons are found in the ventral periaqueductal gray matter, and selective destruction of these DA neurons increases daily sleep time by $20 \%$ [11]. DA is inactivated primarily through reuptake by the DA transporter. Stimulant drugs such as amphetamines and modafinil block the DA transporter and reduce DA reuptake [12], suggesting that the net effect of global elevations in DA signaling is arousal. Several antipsychotic medications block DA receptors and are sedating [5].

DA may play a role in restless legs syndrome (RLS) and the excessive daytime sleepiness associated with RLS. Evidence supporting a role for DA in the pathophysiology of RLS includes improvement in symptoms in response to DA agonist agents and, conversely, worsening RLS symptoms with blockade of DA receptors. However, positron emission tomography (PET) and single-photon emission computed tomography (SPECT) studies imaging DA transporter and receptor densities in human patients with RLS show conflicting results
Table 1 Summary of arousal and sleep regulatory neuronal systems

\begin{tabular}{lllll}
\hline Neurotransmitter & Location & \multicolumn{2}{l}{ Neuronal activity } & \\
\cline { 3 - 4 } & & Wake & NREM & REM \\
\hline Histamine & Tuberomammillary nucleus & $\uparrow \uparrow \uparrow$ & $\uparrow$ & $\leftrightarrow$ \\
Serotonin & Dorsal raphe nucleus & $\uparrow \uparrow \uparrow$ & $\uparrow$ & $\leftrightarrow$ \\
Noradrenalin & Locus coeruleus & $\uparrow \uparrow \uparrow$ & $\uparrow$ & $\leftrightarrow$ \\
Hypocretin/orexin & Lateral hypothalamus & $\uparrow \uparrow \uparrow$ & $\leftrightarrow$ & $\leftrightarrow$ \\
Dopamine & Ventral tegmental area/ventral periaqueductal gray & $\uparrow \uparrow \uparrow$ & $\uparrow$ & $?$ \\
Acetylcholine & Laterodorsal tementum/basal forebrain & $\uparrow \uparrow \uparrow$ & $\uparrow$ & $\uparrow \uparrow \uparrow$ \\
Glutamate & Parabrachial nucleus & $\uparrow \uparrow \uparrow \uparrow$ & $\uparrow$ & $\uparrow \uparrow \uparrow$ \\
GABA & MnPO/VLPO & $\uparrow$ & $\uparrow \uparrow \uparrow$ & $\uparrow \uparrow \uparrow$ \\
GABA & Medullary parafacial zone & $\uparrow$ & $\uparrow \uparrow \uparrow$ & $?$ \\
\hline
\end{tabular}

$\uparrow \uparrow \uparrow$ high $\uparrow$ low $\leftrightarrow$ silent ? unknown 
[13-17]. Potentially relevant neuroanatomical pathways in RLS could involve the A11 diencephalic DA cell cluster, which sends relays to dorsal root ganglion/sensory afferents in the spinal cord $[18,19]$.

Glutamate GLU is a widespread excitatory neurotransmitter in the brain, and GLU-containing neurons are found in the pontine and midbrain reticular formation. Specifically, GLU neurons in the parabrachial/precoeruleus (PB/PC) region of the pons have been implicated in the regulation of cortical arousal. Cell-specific lesions of the $\mathrm{PB} / \mathrm{PC}$ disrupt desynchronized EEG patterns during waking and in response to arousing stimuli [20]. Projections of GLU neurons in the $\mathrm{PB} / \mathrm{PC}$ to cortically projecting neurons in the basal forebrain are important in mediating $\mathrm{PB} / \mathrm{PC}$ effects on cortical activation. Similar to ACH, extracellular levels of GLU in the cortex and in the hypothalamus are elevated during waking and REM sleep compared to NREM sleep [21, 22], suggesting that GLU and $\mathrm{ACH}$ neurotransmission contributes to brain activation in both states.

Hypocretin/Orexin The HCTs are peptides expressed by neurons in the perifornical lateral hypothalamus. HCT neurons have extensive projections to other hypothalamic nuclei, the limbic system, thalamus, and cortex and to the brain stem and spinal cord [23]. HCT neurons occupy a powerful position among arousal systems in that they target the monoaminergic cell groups, ACH neurons in the brain stem and basal forebrain, and DA neurons in the VTA [24, 25]. Acting through HCT-1 and/or HCT-2 receptors, the neuropeptides exert predominately excitatory effects. Given their central position among the arousal circuits, integrity of the HCT system is critical for consolidated arousal states. Knockout of HCT peptides or HCT receptors in mice yields a narcolepsy-like phenotype that includes fragmented wakefulness [26]. Central or systemic administration of dual antagonists of HCT- 1 and HCT-2 receptors has been shown to promote sleep and EEG synchrony in several species [27].

Discharge of HCT neurons is highest during waking, declines dramatically during NREM sleep, and remains low during REM sleep [6]. Discharge during waking is phasic, often occurring during vigorous waking movements. Studies in mice and humans suggest that maximal activity of HCT neurons occurs when working for positive reinforcement (mice) and during positive emotions and social interactions (humans) $[28,29]$.

\section{Neurological Disorders of Arousal}

Several clinical neurological disorders exhibiting deficits in central arousal mechanisms are worth mentioning, namely narcolepsy (with and without cataplexy), but also Alzheimer's disease (AD), Parkinson's disease (PD), and traumatic brain injury (TBI). HCT deficiency or dysfunction may be a common mechanism underlying the inability to maintain normal arousal in these disease states (Table 2).

Patients with narcolepsy experience excessive daytime sleepiness and "sleep attacks." Narcolepsy in humans results from the loss of HCT-producing neurons within the lateral and posterior hypothalami [30]. Recent evidence, including the increase in the incidence of narcolepsy after the H1N1 vaccination in 2010, supports a role for autoimmunity in the destruction of HCT neurons [31, 32]. Of note, in the current International Classification of Sleep Disorders 3rd Edition (ICSD-3), narcolepsy type 1 (formerly known as "narcolepsy with cataplexy" and sometimes referred to as "hypocretin deficiency syndrome") is distinguished from narcolepsy type 2 (formerly known as "narcolepsy without cataplexy") on the basis of HCT deficiency [33]. In addition, the HA system may also be affected in narcolepsy, as HA levels in the CSF may be low in patients with narcolepsy and primary hypersomnia [34]. Two independent groups recently reported an increased number of HA cells in the TMN in post-mortem cases of human narcolepsy, possibly due to a compensatory response to HCT loss and/or related to the process itself causing human narcolepsy [35, 36].

In $\mathrm{AD}$, the most common sleep-related complaints are insomnia, sleep fragmentation, and excessive daytime sleepiness [37]. Neuropathology of the cholinergic basal forebrain and monoaminergic locus coeruleus both feature prominently and early in the time course of $\mathrm{AD}[38,39]$. Given the roles of $\mathrm{ACH}$ and $\mathrm{NE}$ in arousal identified from basic neuroscience studies above, both neurotransmitter systems likely contribute to symptoms of excessive daytime sleepiness in AD. Similarly, recent convergent evidence from several different groups has pointed to an important role for HCT in the pathogenesis of AD. Using a transgenic mouse model of AD, HCT infusion into the brain directly increased interstitial levels of soluble amyloid-beta, and administration of the dual HCT receptor antagonist almorexant significantly reduced amyloid-beta plaque burden [40•]. Double transgenic mice overexpressing

Table 2 Affected arousal and sleep regulatory neuronal systems in selected neurological disorders

\begin{tabular}{llllllll}
\hline Disease & \multicolumn{9}{l}{ Neurotransmitter } & & & \\
\cline { 2 - 7 } & HA & HCT & NA & DA & ACH & GABA \\
\hline Alzheimer's disease & & $\uparrow$ & $\downarrow$ & & $\downarrow$ & $\downarrow$ \\
Parkinson's disease & & $\downarrow$ & & $\downarrow$ & & \\
Traumatic brain injury & $\downarrow$ & $\downarrow$ & & & & \\
Narcolepsy type 1 & $\uparrow$ & $\downarrow$ & & & & \\
Primary hypersomnia & $\downarrow$ & & & & & $\uparrow$
\end{tabular}

$A C H$ acetylcholine, $D A$ dopamine, $G A B A$ gamma-aminobutyric acid, $H C T$ hypocretin (orexin), $H A$ histamine, $N A$ noradrenalin 
amyloid plaques and with a knockout of the HCT gene showed a significant decrease in amyloid plaques along with an increase in total sleep time [41]. Human studies also support the role of HCT in the progression of AD. Increased cerebrospinal fluid (CSF) levels of HCT significantly predict sleep-wake deterioration in $\mathrm{AD}$ patients and are significantly associated with cognitive decline [42•].

In $\mathrm{PD}$, the degeneration of the nigrostriatal DA system is the neuropathological hallmark of the disease. As pharmacological studies of brain DA demonstrate a potent role for this neurotransmitter in regulating generalized brain activation, DA deficiency may contribute to symptoms of excessive daytime sleepiness in PD. Recent studies indicate that HCT deficiency may also contribute to hypersomnia in advanced PD [43-47].

Patients with TBI of all severity types (even mild TBI, without obvious lesions seen on neuroimaging) frequently complain of excessive daytime sleepiness and the inability to maintain normal levels of arousal. The neuropathology related to sleep-wake disturbances in TBI is just beginning to be explored in both animal and human studies. Given the phenotype of excessive daytime sleepiness and nighttime sleep fragmentation in human TBI which mimics narcolepsy, several studies have examined HCT in TBI. Measured HCT levels in the cerebrospinal fluid (CSF) were low in $95 \%$ of 44 patients within the first 4 days of moderate to severe TBI [48]. A small study of four patients who died 7 to 42 days after severe TBI showed a $27 \%$ reduction in the number of HCT neurons compared to non-TBI controls [49]. Likewise, studies using mouse models of mild [50•] and moderate [51] TBI show decreased brain HCT levels and decreased HCT neuron activation during wakefulness after brain injury. However, deficits in HCT are unlikely to explain all sleep-wake abnormalities, especially in the chronic phase of TBI, as CSF HCT levels for the most part return to baseline after 6 months post-TBI [52]. In support of this, a recent post-mortem human study in 12 patients with severe TBI showed a $41 \%$ loss of histaminergic neurons in the tuberomammillary nucleus, compared to control subjects [53].

These findings implicating $\mathrm{ACH}$, monoamines, $\mathrm{DA}$, and HCT in clinical disorders of arousal such as narcolepsy, AD, $\mathrm{PD}$, and TBI strongly support the basic neuroscience data described above.

\section{Sleep Onset and NREM Sleep-Promoting Mechanisms}

Brain mechanisms that promote sleep must be able to facilitate a coordinated suppression of activity in multiple groups of arousal-promoting neurons over a period of seconds to minutes at the wake to sleep transition, and maintain that suppression of activity over the subsequent sleep period lasting many minutes (rodents) to hours (primates). The onset and maintenance of sleep are accomplished through interactions among three cellular/molecular and neurochemical mechanisms: (1) systems of GABAergic sleep-promoting neurons located in the preoptic hypothalamus and rostral medulla that exert inhibitory control over key arousal systems, (2) production of endogenous sleep regulatory substances during waking that regulate homeostatic sleep drive by targeting both wakeand sleep regulatory circuits in multiple brain regions, and (3) output of the circadian clock in the suprachiasmatic nucleus that functions to promote wakefulness and sleep at different times of day. The complex topic of the circadian control of sleep and its role in sleep disturbance in neurological disorders is beyond the scope of this article, and the reader is referred to recent comprehensive reviews of the subject [54-56].

Preoptic Hypothalamic Neurons Sleep regulatory neurons have been identified by direct neuronal recording during sleep and wakefulness [57] and by sleep-related expression of the protein product of the $c-f o s$ gene, an anatomical marker of neuronal activity [58]. The functional importance of sleepactive neurons is confirmed by loss of function lesion studies. These approaches have identified two subregions of the preoptic hypothalamus that contain high densities of functionally important sleep-active neurons, the ventrolateral preoptic area (VLPO) $[58,59]$ and the median preoptic nucleus (MnPO) [60]. VLPO and MnPO neurons exhibit elevated discharge rates during NREM and REM sleep compared to waking (Table 1). MnPO and VLPO neurons are dynamically responsive to changes in homeostatic sleep pressure induced by sleep deprivation [61•].

Sleep-related c-Fos expression is co-localized with GABA in the MnPO [60] and with GABA and galanin in the VLPO $[58,62]$. VLPO neurons project heavily to HA neurons in the TMN and to the DRN, LC, and the PPT/LDT [63]. MnPO neurons project to the DRN and LC. MnPO and VLPO neurons that express sleep-related c-Fos project to the HCT neuronal field in the lateral hypothalamus [64]. Sleep-active neurons in VLPO and MnPO exhibit discharge profiles across the wake-NREM-REM cycle that are reciprocal to those of wakepromoting HA, 5HT, NE, and HCT neurons [63] . These findings support the hypothesis that VLPO/MnPO neurons promote sleep through monosynaptic GABAergic inhibition of monoaminergic, cholinergic, and hypocretinergic neurons. GABAergic VLPO neurons are inhibited by ACH, 5HT, and $\mathrm{NE}$, and reciprocal inhibitory interactions between sleep and arousal regulatory neurons are hypothesized to function as a bi-stable sleep-wake switch [65].

Rostral Medullary Neurons An older literature examining the behavioral and EEG effects of brain stem transections and lesions provided evidence for sleep-promoting mechanisms in the brain stem as well as the forebrain [66]. Recent findings 
have identified a sleep regulatory neuronal group in the rostral medulla of rats and mice, located lateral and dorsal to the facial nerve in the parafacial zone (PZ) [67, 68•]. PZ neurons express c-Fos during sleep but not during waking and sleeprelated c-Fos is co-localized with markers for GABA [67]. Excitotoxic lesions of the PZ result in persistent sleep loss [67], and acute pharmacogenetic activation of PZ GABAergic neurons in transgenic mice results in increases in non-REM sleep and suppression of waking and REM sleep [68 ${ }^{\bullet}$. PZ neurons project rostrally to the $\mathrm{PB} / \mathrm{PC}$ nuclei [67] that contain arousal-promoting GLU neurons [20]. Optogenetic activation of GABAergic neurons in the PZ evokes GABA-mediated inhibition of $\mathrm{PB} / \mathrm{PC}$ neurons that project to the basal forebrain [68•]. The ascending circuit of GABAergic PZ neurons evoking sleep-related inhibition of $\mathrm{PB} / \mathrm{PC}$ neurons that normally excites cortically projecting basal forebrain neurons during waking complements the descending sleep-related inhibition of arousal systems that originates in the MnPO/VLPO.

Sleep Regulatory Substances Homeostatic sleep pressure accumulates slowly during periods of sustained waking and dissipates slowly during subsequent sleep. It has long been hypothesized that accumulation and dissipation of endogenous somnogenic substances during waking and sleep, respectively, underlie the dynamics of sleep homeostasis [69].

Adenosine (ADO) is an inhibitory neuromodulator in the CNS, whose role in sleep is suggested by the potent arousalproducing effects of caffeine, an antagonist of $A_{1}$ and $A_{2 A}$ ADO receptors [70]. Sleep deprivation is accompanied by elevated ADO levels in the basal forebrain and cortex, followed by a decline during recovery sleep [4, 71]. ADO and its analogs promote sleep after systemic and central administration, and ADO-induced sleep is accompanied by increased EEG slow-wave activity (SWA), as is sleep that follows sleep deprivation $[4,70]$. Astrocytes as well as neurons are sources of $\mathrm{ADO}$ in the brain. A conditional knockout of the $\mathrm{A}_{1}-\mathrm{R}$ gene in mice attenuates EEG SWA in NREM sleep following sleep deprivation [72]. ACH neurons in the basal forebrain are important targets of the sleep-promoting effects of ADO acting on $A_{1} R$ [73]. Additional arousal systems may be targeted by ADO. Local perfusion of $A_{1}-\mathrm{R}$ agonists in the rat lateral hypothalamus suppresses waking c-Fos expression in HCT neurons [74]. Complementary actions of ADO on the excitatory $A_{2 A}-R$ also impact sleep. Central administration of $A_{2 A}-R$ agonists promotes sleep and activates VLPO neurons [75]. Administration of $\mathrm{A}_{2 \mathrm{~A}}$ agonists into the subarachnoid space ventral to the preoptic area increases sleep and increases c-Fos expression in GABAergic neurons in the MnPO and VLPO [76]. $A_{2 A}$ receptors in the shell of the nucleus accumbens have also been implicated in sleep regulation [9].

Cytokines, including interleukin- $1 \beta$ (IL-1 $\beta$ ) and tumor necrosis factor- $\alpha$ (TNF- $\alpha$ ), are sleep promoting [69]. Antagonism of IL- $1 \beta$ and TNF- $\alpha$ can disrupt normal sleep and impair homeostatic responses to sleep deprivation $[69,77]$. Expression of IL- $1 \beta$ and TNF- $\alpha$ is elevated in multiple brain regions in response to waking neuronal activity [77]. Cellular mechanisms of cytokine-mediated sleep generation are not completely understood but may involve a combination of arousal system inhibition and activation of preoptic sleep regulatory neurons [78-81].

\section{Neurological Disorders of Sleep and Sleep-Promoting Systems}

Several clinical disorders also involve the VLPO, GABA, and galanin systems as described above. We discuss two such examples below for Alzheimer's disease (AD) and primary hypersomnia (Table 2). These examples highlight the fact that sleep-promoting systems identified in animal models are highly conserved and that inhibitory pathways within the hypothalamus may also be relevant to human neurological disorders of sleep and excessive sleepiness.

Patients with $\mathrm{AD}$ frequently report significant insomnia and sleep fragmentation. A recent study examining 45 older adults with and without $\mathrm{AD}$ found that individuals with more galanin-immunoreactive neurons within the intermediate nucleus (the putative human homologue to VLPO in rodents) exhibited less fragmented sleep [82]. This suggests that GABA/galanin in the intermediate nucleus is important for the consolidation of sleep and that clinical disorders exhibiting sleep fragmentation may involve this neuroanatomical pathway.

Patients with primary hypersomnia have excessive daytime sleepiness in the absence of other known causes of sleepiness. A recent study in 32 hypersomnolent patients identified a naturally occurring substance in human CSF which augmented inhibitory GABA signaling in vitro - though the exact substance is still yet to be determined. Furthermore, flumazenil (a GABAergic benzodiazepine receptor antagonist) normalized vigilance in a subset of these patients [83•]. The same group has also studied the macrolide antibiotic clarithromycin in enhancing vigilance in hypersomnia patients, presumably via pharmacological antagonism of GABA-A receptors. A retrospective review of clarithromycin treatment in 53 patients with this GABA-related hypersomnia showed $64 \%$ reported improvement in daytime sleepiness [84]. These findings suggest that GABA may play a role in the promotion of sleep in pathological hypersomnia. However, as mentioned in the "Neurological Disorders of Arousal" section, patients with primary hypersomnia can also have low levels of CSF histamine, although this remains somewhat controversial [34, 85]. Therefore, the pathophysiology of hypersomnia is likely to be complex and multifactorial in nature. 


\section{REM Sleep}

Muscle Atonia The core circuits that generate the REM sleep state are located in the brain stem. The most striking manifestation of REM sleep is the coincidence of widespread brain activation with somatic muscle atonia. Several of the waking neuronal arousal systems are silent during REM sleep, with activation of wake-REM active GLU and ACH neurons supporting brain activation during REM sleep. At the level of motor neurons in the spinal cord, REM sleep muscle atonia is the result of GABA and glycine-mediated postsynaptic inhibition, indicated by motoneuron hyperpolarization and episodic inhibitory postsynaptic potentials (IPSPs). Sources of inhibition are spinally projecting GABA/glycine neurons in the ventromedial medulla and glycinergic spinal interneurons. Hyperpolarization and episodic IPSPS have been recently described in hypoglossal motoneurons during REM sleep [86]. Disfacilitation of brain stem and spinal cord motoneurons contributes to REM sleep atonia due to loss of excitatory inputs from 5HT, NA, and HCT neurons. REM sleep atonia is periodically interrupted by phasic muscle twitches that often accompany bursts of rapid eye movements. Phasic twitches are associated with GLU-mediated excitatory postsynaptic potentials in motoneurons and reflect activation of central motor systems during REM sleep.

REM Sleep-Generating Mechanisms An older literature, based largely on electrophysiological and neuropharmacological studies in cats, emphasized ACH neurons in the PPT/LDT and their reciprocal inhibitory interactions with monoaminergic neurons as being critical for the switching between nonREM to REM sleep [70]. Recent findings in rodents emphasize interactions among GABAergic and glutamatergic neurons in the caudal midbrain and pons. A population of "REM on" neurons (i.e., neurons with elevated discharge during REM sleep compared to waking and NREM sleep) is located ventral to the LC $[7,87,88]$. This part of the rostral pons has been variously called subcoeruleus nucleus, pontine inhibitory area, and peri-LC $\alpha$, but the sublaterodorsal tegmental nucleus (SLD) is currently most widely used. In addition to the SLD, expression of c-Fos during augmented REM sleep in rats has identified REM on neurons in the precoeruleus region and medial parabrachial nucleus $[89,90]$. REM off GABAergic neurons in the ventrolateral periaqueductal gray (vlPAG) and lateral pontine tegmentum (LPT) inhibit the activity of REM on neurons in the SLD [91]. One model of NREM-REM switching involves reciprocal inhibitory interactions among GABAergic vlPAG/LPT neurons and GABAergic REM on neurons in the SLD [65]. The ventral SLD contains glutamatergic REM on neurons that project to GABA/glycine neurons in the ventromedial medulla and spinal cord and regulates muscle atonia of REM sleep [88, 91]. Cell-specific lesions or inhibition of the vlPAG/LPT increases the amount of REM sleep and produce cataplexy-like periods of atonia during wakefulness, and SLD lesions and medial medulla lesions can result in REM sleep without atonia [88]. Another set of GLU neurons in the PB/PC nuclei project to the basal forebrain and regulate EEG phenomena of REM sleep. Current evidence suggests that most ACH neurons in the LDT/PPT are wake/REM active [7] and, through ascending projections, promote thalamocortical desynchrony during REM sleep. $\mathrm{ACH}$ in the LDT/PPT and monoaminergic neurons in the TMN, DRN, and LC appear to be modulatory of the core REM-generating circuitry, with ACH signaling facilitating REM sleep and monoaminergic neuronal activation suppressing REM sleep.

Hypothalamic Modulation of REM Sleep Descending inputs from sleep and arousal regulatory hypothalamic neuronal systems are sources of modulatory control of REM sleep circuits. The HCT peptides have REM-suppressing effects. HCT receptor antagonists augment REM sleep [27]. Optogenetic activation of HCT neurons during either NREM or REM sleep evokes waking [92]. HCT neurons target key nodes in brain stem REM sleep circuitry including neurons in the vlPAG/LPT, DRN, and LC. Activation of HCT neurons during waking suppresses manifestations of REM sleep, and REM-generating circuits are disinhibited during NREM sleep when HCT neuronal activity is minimal.

Neurons expressing the inhibitory peptide melaninconcentrating hormone $(\mathrm{MCH})$ are localized in the lateral hypothalamus, zona incerta, and dorsomedial hypothalamus [93]. Included among the targets of MCH neurons are LC, DRN, and vlPAG. MCH neurons also express GABA. MCH neurons are nearly silent during waking, seldom discharge during NREM sleep, and are most active during REM sleep [94]. Expression of c-Fos in $\mathrm{MCH}$ neurons is increased during REM-enriched sleep following prolonged REM sleep deprivation [95]. Optogenetic activation of $\mathrm{MCH}$ neurons during NREM sleep significantly increases the probability of transitions to REM sleep [96]. Mechanisms of MCH REM sleep enhancement involve disinhibition of SLD REM on neurons via $\mathrm{MCH} / \mathrm{GABA}$ inhibition of REM off neurons in the TMN, vlPAG, DRN, and LC [93, 96].

GABAergic neurons in the VLPO target many of the REM off neuronal populations that impact the SLD, including the vlPAG, DRN, and LC [63]. The majority of VLPO neurons exhibit elevated discharge during both NREM and REM sleep, and a population of REM on neurons is located in the dorsal extended VLPO [57, 61•]. Similar to $\mathrm{MCH}$ neurons, inhibitory effects of VLPO neurons on the REM off neuronal populations facilitate REM sleep onset and maintenance through disinhibition of the SLD. 


\section{Neurological Disorders of REM Sleep}

Several clinical disorders are associated with REM sleep behavior disorder (RBD), which involves loss of REM sleep muscle atonia. The most common neurological disorders include the alpha-synucleinopathies, such as PD and "Parkinson's plus" syndromes like dementia with Lewy bodies (DLB) and multiple systems atrophy. The neuropathology in these synucleinopathies largely supports findings in animal models that suggest the SLD and other brain stem regions are critical for normal REM sleep atonia.

Neuroimaging studies show that damage within or near the pontine tegmental regions (which includes the SLD) is associated with RBD symptoms [97-99]. A recent imaging study in 36 human subjects with PD showed that the degree of neuromelanin signal (a surrogate marker for neuronal integrity) in the SLD strongly predicted REM sleep without atonia in PD patients with RBD, but not in PD patients without RBD [100]. Furthermore, pathological neurodegeneration characteristic of PD and Parkinson's plus syndromes (i.e., Lewy bodies, gliosis, neuronal loss) is frequently observed in or near the subcoeruleus, gigantocellular reticular nucleus, and pedunculopontine nucleus $[101,102]$. These findings support data from animal models implicating the SLD and other brain stem regions in $\mathrm{RBD}$.

\section{Conclusions}

Basic research using animal models of arousal and sleep mechanisms continues to identify novel neuroanatomical circuits controlling sleep and wakefulness in human clinical disorders. Arousal mechanisms include histamine (HA), serotonin (5HT), noradrenaline (NA), acetylcholine (ACH), glutamate (GLU), dopamine (DA), and hypocretin/orexin (HCT) as neurotransmitters acting within the basal forebrain, hypothalamus, and brain stem. Clinical disorders of arousal including narcolepsy, Alzheimer's disease, Parkinson's disease, and traumatic brain injury appear to involve HCT and possibly histamine as common mechanisms related to excessive daytime sleepiness. NREM sleep-promoting pathways largely involve GABA and galanin in the VLPO and MnPO and show striking similarity between phenotypes in rodent models and human disorders such as Alzheimer's disease and primary hypersomnia. REM sleep-promoting pathways, especially those involving the SLD of the pons, also show parallels between rodent models of REM muscle atonia and human RBD associated with Parkinson's disease and other synucleinopathies. In conclusion, our understanding of the basic neurobiology of arousal and sleep-promoting systems continues to evolve with the use of animal models to inform further studies on human clinical sleep disorders and vice versa.
Acknowledgments This material is the result of work supported with resources and the use of facilities at the VA Portland Health Care System and the VA Career Development Award \# IK2 BX002712 (MML) and the VA Greater Los Angeles Healthcare System (RS).

\section{Compliance with Ethics Guidelines}

Conflict of Interest Miranda M. Lim and Ronald Szymusiak declare that they have no conflict of interest.

Human and Animal Rights and Informed Consent This article does not contain any studies with human or animal subjects performed by any of the authors.

\section{References}

Papers of particular interest, published recently, have been highlighted as:

- Of importance

1. Lin JS, Anaclet C, Sergeeva OA, Haas HL. The waking brain: an update. Cell Mol Life Sci: CMLS. 2011;68(15):2499-512.

2. Haas HL, Sergeeva OA, Selbach O. Histamine in the nervous system. Physiol Rev. 2008;88(3):1183-241.

3. Monti JM. Serotonin control of sleep-wake behavior. Sleep Med Rev. 2011;15(4):269-81.

4. Brown RE, Basheer R, McKenna JT, Strecker RE, McCarley RW. Control of sleep and wakefulness. Physiol Rev. 2012;92(3):1087187.

5. Espana RA, Scammell TE. Sleep neurobiology from a clinical perspective. Sleep. 2011;34(7):845-58.

6. Jones BE. Modulation of cortical activation and behavioral arousal by cholinergic and orexinergic systems. Ann N Y Acad Sci. 2008;1129:26-34.

7. Boucetta S, Cisse Y, Mainville L, Morales M, Jones BE. Discharge profiles across the sleep-waking cycle of identified cholinergic, GABAergic, and glutamatergic neurons in the pontomesencephalic tegmentum of the rat. J Neurosci. 2014;34(13):4708-27.

8. Irmak SO, de Lecea L. Basal forebrain cholinergic modulation of sleep transitions. Sleep. 2014;37(12):1941-51.

9. Lazarus M, Huang ZL, Lu J, Urade Y, Chen JF. How do the basal ganglia regulate sleep-wake behavior? Trends Neurosci. 2012;35(12):723-32.

10. Qiu MH, Yao QL, Vetrivelan R, Chen MC, Lu J. Nigrostriatal dopamine acting on globus pallidus regulates sleep. New York: Cerebral cortex; 2014

11. Lu J, Jhou TC, Saper CB. Identification of wake-active dopaminergic neurons in the ventral periaqueductal gray matter. $\mathrm{J}$ Neurosci: Off J Soc Neurosci. 2006;26(1):193-202.

12. Wisor J. Modafinil as a catecholaminergic agent: empirical evidence and unanswered questions. Front Neurol. 2013;4:139.

13. Earley CJ, Kuwabara H, Wong DF, Gamaldo C, Salas RE, Brasic $\mathrm{JR}$, et al. Increased synaptic dopamine in the putamen in restless legs syndrome. Sleep. 2013;36(1):51-7.

14. Earley CJ, Kuwabara H, Wong DF, Gamaldo C, Salas R, Brasic J, et al. The dopamine transporter is decreased in the striatum of subjects with restless legs syndrome. Sleep. 2011;34(3):341-7.

15. Cervenka S, Palhagen SE, Comley RA, Panagiotidis G, Cselenyi $\mathrm{Z}$, Matthews JC, et al. Support for dopaminergic hypoactivity in 
restless legs syndrome: a PET study on D2-receptor binding. Brain: J Neurol. 2006;129(Pt 8):2017-28.

16. Tribl GG, Asenbaum S, Happe S, Bonelli RM, Zeitlhofer J, Auff E. Normal striatal D2 receptor binding in idiopathic restless legs syndrome with periodic leg movements in sleep. Nucl Med Commun. 2004;25(1):55-60.

17. Michaud M, Soucy JP, Chabli A, Lavigne G, Montplaisir J. SPECT imaging of striatal pre- and postsynaptic dopaminergic status in restless legs syndrome with periodic leg movements in sleep. J Neurol. 2002;249(2):164-70.

18. Earley CJ, Allen RP, Connor JR, Ferrucci L, Troncoso J. The dopaminergic neurons of the A11 system in RLS autopsy brains appear normal. Sleep Med. 2009;10(10):1155-7.

19. Rye DB. The two faces of eve: dopamine's modulation of wakefulness and sleep. Neurology. 2004;63(8 Suppl 3):S2-7.

20. Fuller PM, Sherman D, Pedersen NP, Saper CB, Lu J. Reassessment of the structural basis of the ascending arousal system. J Comp Neurol. 2011;519(5):933-56.

21. Dash MB, Douglas CL, Vyazovskiy VV, Cirelli C, Tononi G. Long-term homeostasis of extracellular glutamate in the rat cerebral cortex across sleep and waking states. J Neurosci: Off J Soc Neurosci. 2009;29(3):620-9.

22. John J, Ramanathan L, Siegel JM. Rapid changes in glutamate levels in the posterior hypothalamus across sleep-wake states in freely behaving rats. Am J Physiol Regul, Int Comp Physiol. 2008;295(6):R2041-9.

23. Peyron C, Tighe DK, van Den Pol AN, de Lecea L, Heller HC, Sutcliffe JG, et al. Neurons containing hypocretin (orexin) project to multiple neuronal systems. J Neurosci. 1998;18:9996-10015.

24. de Lecea L. Hypocretins and the neurobiology of sleep-wake mechanisms. Prog Brain Res. 2012;198:15-24.

25. Saper CB, Fuller PM, Pedersen NP, Lu J, Scammell TE. Sleep state switching. Neuron. 2011;68(6):1023-42.

26. Burgess CR, Scammell TE. Narcolepsy: neural mechanisms of sleepiness and cataplexy. J Neurosci. 2012;32(36):12305-11.

27. Winrow CJ, Renger JJ. Discovery and development of orexin receptor antagonists as therapeutics for insomnia. $\mathrm{Br} \mathrm{J}$ Pharmacol. 2014;171(2):283-93.

28. McGregor R, Wu MF, Barber G, Ramanathan L, Siegel JM. Highly specific role of hypocretin (orexin) neurons: differential activation as a function of diurnal phase, operant reinforcement versus operant avoidance and light level. J Neurosci. 2011;31(43): 15455-67.

29. Blouin AM, Fried I, Wilson CL, Staba RJ, Behnke EJ, Lam HA, et al. Human hypocretin and melanin-concentrating hormone levels are linked to emotion and social interaction. Nat Commun. 2013;4:1547.

30. Thannickal TC, Moore RY, Nienhuis R, Ramanathan L, Gulyani $\mathrm{S}$, Aldrich $\mathrm{M}$, et al. Reduced number of hypocretin neurons in human narcolepsy. Neuron. 2000;27(3):469-74.

31. Arango MT, Kivity S, Shoenfeld Y: Is narcolepsy a classical autoimmune disease? Pharmacol Res: Off J Ital Pharmacol Soc. 2014.

32. Partinen M, Kornum BR, Plazzi G, Jennum P, Julkunen I, Vaarala O. Narcolepsy as an autoimmune disease: the role of H1N1 infection and vaccination. Lancet Neurol. 2014;13(6):600-13.

33. International classification of sleep disorders. American Academy of Sleep Medicine, Darien. 2014.

34. Bassetti CL, Baumann CR, Dauvilliers Y, Croyal M, Robert P, Schwartz JC. Cerebrospinal fluid histamine levels are decreased in patients with narcolepsy and excessive daytime sleepiness of other origin. J Sleep Res. 2010;19(4):620-3.

35. Valko PO, Gavrilov YV, Yamamoto M, Reddy H, Haybaeck J, Mignot E, et al. Increase of histaminergic tuberomammillary neurons in narcolepsy. Ann Neurol. 2013;74(6):794-804.
36. John J, Thannickal TC, McGregor R, Ramanathan L, Ohtsu H, Nishino S, et al. Greatly increased numbers of histamine cells in human narcolepsy with cataplexy. Ann Neurol. 2013;74(6):786-93.

37. Guarnieri B, Adorni F, Musicco M, Appollonio I, Bonanni E, Caffarra $\mathrm{P}$, et al. Prevalence of sleep disturbances in mild cognitive impairment and dementing disorders: a multicenter Italian clinical cross-sectional study on 431 patients. Dement Geriatr Cogn Disord. 2012;33(1):50-8.

38. Weinshenker D. Functional consequences of locus coeruleus degeneration in Alzheimer's disease. Curr Alzheimer Res. 2008;5(3):342-5.

39. Whitehouse PJ, Price DL, Struble RG, Clark AW, Coyle JT, Delon MR. Alzheimer's disease and senile dementia: loss of neurons in the basal forebrain. Science. 1982;215(4537):1237-9.

40. Kang JE, Lim MM, Bateman RJ, Lee JJ, Smyth LP, Cirrito JR, et al. Amyloid-beta dynamics are regulated by orexin and the sleep-wake cycle. Science. 2009;326(5955):1005-7. First study to show that orexin (hypocretin) directly modulates amyloid beta levels and amyloid plaque deposition using a transgenic mouse model of $\mathrm{AD}$.

41. Roh JH, Jiang H, Finn MB, Stewart FR, Mahan TE, Cirrito JR, Heda A, Snider BJ, Li M, Yanagisawa M, de Lecea L et al.: Potential role of orexin and sleep modulation in the pathogenesis of Alzheimer's disease. J Exp Med. 2014.

42. Liguori C, Romigi A, Nuccetelli M, Zannino S, Sancesario G, Martorana A, et al. Orexinergic system dysregulation, sleep impairment, and cognitive decline in Alzheimer disease. JAMA Neurol. 2014;71(12):1498-505. This recent study in human AD patients identifies a role for CSF hypocretin in sleepwake disturbances and cognitive impairment.

43. Knie B, Mitra MT, Logishetty K, Chaudhuri KR. Excessive daytime sleepiness in patients with Parkinson's disease. CNS Drugs. 2011;25(3):203-12.

44. Fronczek R, Baumann CR, Lammers GJ, Bassetti CL, Overeem S. Hypocretin/orexin disturbances in neurological disorders. Sleep Med Rev. 2009;13(1):9-22.

45. Verbaan D, van Rooden SM, Visser M, Marinus J, van Hilten JJ. Nighttime sleep problems and daytime sleepiness in Parkinson's disease. Mov Disord: Off J Mov Disord Soc. 2008;23(1):35-41.

46. Thannickal TC, Lai YY, Siegel JM. Hypocretin (orexin) cell loss in Parkinson's disease. Brain: J Neurol. 2007;130(Pt 6):1586-95.

47. Fronczek R, Overeem S, Lee SY, Hegeman IM, van Pelt J, van Duinen SG, et al. Hypocretin (orexin) loss in Parkinson's disease. Brain: J Neurol. 2007;130(Pt 6):1577-85.

48. Baumann CR, Stocker R, Imhof HG, Trentz O, Hersberger M, Mignot E, et al. Hypocretin-1 (orexin A) deficiency in acute traumatic brain injury. Neurology. 2005;65(1):147-9.

49. Baumann CR, Bassetti CL, Valko PO, Haybaeck J, Keller M, Clark E, et al. Loss of hypocretin (orexin) neurons with traumatic brain injury. Ann Neurol. 2009;66(4):555-9.

50. Lim MM, Elkind J, Xiong G, Galante R, Zhu J, Zhang L, et al. Dietary therapy mitigates persistent wake deficits caused by mild traumatic brain injury. Sci Transl Med. 2013;5(215):215ra173. This recent study in human AD patients identifies a role for CSF hypocretin in sleep-wake disturbances and cognitive impairment.

51. Lim MM, Willie JT, Bennett RE, Azarion AA, Schwetye KE, Brody DL. Controlled cortical impact traumatic brain injury acutely disrupts wakefulness and extracellular orexin dynamics as determined by intracerebral microdialysis in mice. J Neurotrauma. 2012;29(10):1908-21.

52. Baumann CR, Werth E, Stocker R, Ludwig S, Bassetti CL. Sleepwake disturbances 6 months after traumatic brain injury: a prospective study. Brain: J Neurol. 2007;130(Pt 7):1873-83.

53. Valko PO, Gavrilov YV, Yamamoto M, Finn K, Reddy H, Haybaeck J, Weis S, Scammell TE, Baumann CR: Damage to 
histaminergic tuberomammillary neurons and other hypothalamic neurons with traumatic brain injury. Ann Neurol. 2014.

54. Saper CB. The central circadian timing system. Curr Opin Neurobiol. 2013;23(5):747-51.

55. Kim MJ, Lee JH, Duffy JF. Circadian rhythm sleep disorders. J Clin Outcomes Manag: JCOM. 2013;20(11):513-28.

56. Videnovic A, Lazar AS, Barker RA, Overeem S. 'The clocks that time us' - circadian rhythms in neurodegenerative disorders. Nat Rev Neurol. 2014;10(12):683-93.

57. Szymusiak R, Alam N, Steininger TL, McGinty D. Sleep-waking discharge patterns of ventrolateral preoptic/anterior hypothalamic neurons in rats. Brain Res. 1998;803:178-88.

58. Sherin JE, Elmquist JK, Torrealba F, Saper CB. Innervation of histaminergic tuberomammillary neurons by GABAergic and galaninergic neurons in the ventrolateral preoptic nucleus of the rat. J Neurosci. 1998;18:4705-21.

59. Sherin JE, Shiromani PJ, McCarley RW, Saper CB. Activation of ventrolateral preoptic neurons during sleep. Science. 1996;271: 216-9.

60. Gong H, McGinty D, Guzman-Marin R, Chew KT, Stewart D, Szymusiak R. Activation of c-fos in GABAergic neurones in the preoptic area during sleep and in response to sleep deprivation. J Physiol. 2004;556:935-46.

61. Alam MA, Kumar S, McGinty D, Alam MN, Szymusiak R. Neuronal activity in the preoptic hypothalamus during sleep deprivation and recovery sleep. J Neurophysiol. 2014;111(2):287-99. First demonstration that sleep-regulatory neurons in the preoptic area are dynamically responsive to changing homeostatic sleep pressure.

62. Gaus SE, Strecker RE, Tate BA, Parker RA, Saper CB. Ventrolateral preoptic nucleus contains sleep-active, galaninergic neurons in multiple mammalian species. Neuroscience. 2002;115: 285-94.

63. Szymusiak R, McGinty D. Hypothalamic regulation of sleep and arousal. Ann N Y Acad Sci. 2008;1129:275-86.

64. Uschakov A, Gong H, McGinty D, Szymusiak R. Sleep-active neurons in the preoptic area project to the hypothalamic paraventricular nucleus and the perifornical lateral hypothalamus. Eur J Neurosci. 2006;23:3284-96.

65. Saper CB, Fuller PM, Pedersen NP, Lu J, Scammell TE. Sleep state switching. Neuron. 2010;68(6):1023-42.

66. Villablanca JR. Counterpointing the functional role of the forebrain and of the brainstem in the control of the sleep-waking system. J Sleep Res. 2004;13(3):179-208.

67. Anaclet C, Lin JS, Vetrivelan R, Krenzer M, Vong L, Fuller PM, et al. Identification and characterization of a sleep-active cell group in the rostral medullary brainstem. J Neurosci. 2012;32(50):17970-6.

68. Anaclet C, Ferrari L, Arrigoni E, Bass CE, Saper CB, Lu J, et al. The GABAergic parafacial zone is a medullary slow wave sleeppromoting center. Nat Neurosci. 2014;17(9):1217-24. Identified GABAergic sleep regulatory neurons in the rostral medulla and describes connections with arousal promoting glutamatergic neurons in the pontine parabrachial nucleus.

69. Obal Jr F, Krueger JM. Biochemical regulation of non-rapid-eyemovement sleep. Front Biosci. 2003;8:d520-50.

70. McCarley RW. Neurobiology of REM and NREM sleep. Sleep Med. 2007;8:302-30.

71. Basheer R, Strecker RE, Thakkar MM, McCarley RW. Adenosine and sleep-wake regulation. Prog Neurobiol. 2004;73(6):379-96.

72. Bjorness TE, Kelly CL, Gao T, Poffenberger V, Greene RW. Control and function of the homeostatic sleep response by adenosine A1 receptors. J Neurosci. 2009;29(5):1267-76.

73. Kalinchuk AV, Porkka-Heiskanen T, McCarley RW, Basheer R: Cholinergic neurons of the basal forebrain mediate biochemical and electrophysiological mechanisms underlying sleep homeostasis. Eur J Neurosci. 2014.

74. Rai S, Kumar S, Alam MA, Szymusiak R, McGinty D, Alam MN A1 receptor mediated adenosinergic regulation of perifornicallateral hypothalamic area neurons in freely behaving rats. Neuroscience. 2010;167(1):40-8.

75. Scammell T, Gerashchenko D, Mochizuki T, McCarthy MT, Estabrooke IV, Sears C, et al. An adenosine A2a agonist increases sleep and induces Fos in ventrolateral preoptic area neurons. Neuroscience. 2001;107(653):663.

76. Kumar S, Rai S, Hsieh KC, McGinty D, Alam MN, Szymusiak R. Adenosine $\mathrm{A}(2 \mathrm{~A})$ receptors regulate the activity of sleep regulatory GABAergic neurons in the preoptic hypothalamus. Am J Physiol Regul, Integr Comp Physiol. 2013;305(1):R31-41.

77. Krueger JM, Rector DM, Roy S, Van Dongen HP, Belenky G, Panksepp J. Sleep as a fundamental property of neuronal assemblies. Nat Rev Neurosci. 2008;9(12):910-9.

78. Brambilla D, Franciosi S, Opp MR, Imeri L. Interleukin-1 inhibits firing of serotonergic neurons in the dorsal raphe nucleus and enhances GABAergic inhibitory post-synaptic potentials. Eur J Neurosci. 2007;26(7):1862-9.

79. Brambilla D, Barajon I, Bianchi S, Opp MR, Imeri L. Interleukin1 inhibits putative cholinergic neurons in vitro and REM sleep when microinjected into the rat laterodorsal tegmental nucleus. Sleep. 2010;33(7):919-29.

80. Baker FC, Shah S, Stewart D, Angara C, Gong H, Szymusiak R, et al. Interleukin 1beta enhances non-rapid eye movement sleep and increases c-Fos protein expression in the median preoptic nucleus of the hypothalamus. Am J Physiol Regul Integr Comp Physiol. 2005;288(4):R998-1005.

81. Alam MN, McGinty D, Bashir T, Kumar S, Imeri L, Opp MR, et al. Interleukin-1 beta modulates state-dependent discharge activity of preoptic area and basal forebrain neurons: role in sleep regulation. Eur J Neurosci. 2004;20(1):207-16.

82. Lim AS, Ellison BA, Wang JL, Yu L, Schneider JA, Buchman AS, et al. Sleep is related to neuron numbers in the ventrolateral preoptic/intermediate nucleus in older adults with and without Alzheimer's disease. Brain: A J Neurol. 2014;137(Pt 10):284761.

83. Rye DB, Bliwise DL, Parker K, Trotti LM, Saini P, Fairley J, et al. Modulation of vigilance in the primary hypersomnias by endogenous enhancement of GABAA receptors. Sci Transl Med. 2012;4(161):161 ra151. Identified a novel GABA-like substance in the CSF of patients with primary hypersomnia.

84. Trotti LM, Saini P, Freeman AA, Bliwise DL, Garcia PS, Jenkins A, et al. Improvement in daytime sleepiness with clarithromycin in patients with GABA-related hypersomnia: clinical experience. J Psychopharmacol. 2013;28(7):697-702.

85. Dauvilliers Y, Delallee N, Jaussent I, Scholz S, Bayard S, Croyal $\mathrm{M}$, et al. Normal cerebrospinal fluid histamine and telemethylhistamine levels in hypersomnia conditions. Sleep. 2012;35(10):1359-66.

86. Fung SJ, Chase MH: Postsynaptic inhibition of hypoglossal motoneurons produces atonia of the genioglossal muscle during rapid eye movement sleep. Sleep. 2014.

87. Verret L, Leger L, Fort P, Luppi PH. Cholinergic and noncholinergic brainstem neurons expressing Fos after paradoxical (REM) sleep deprivation and recovery. Eur J Neurosci. 2005;21:2488-504.

88. Peever J, Luppi PH, Montplaisir J. Breakdown in REM sleep circuitry underlies REM sleep behavior disorder. Trends Neurosci. 2014;37(5):279-88.

89. Boissard R, Gervasoni D, Schmidt MH, Barbagli B, Fort P, Luppi $\mathrm{PH}$. The rat ponto-medullary network responsible for paradoxical sleep onset and maintenance: a combined microinjection and 
functional neuroanatomical study. Eur JnNurosci. 2002;16(10): 1959-73.

90. Lu J, Sherman D, Devor M, Saper CB. A putative flip-flop switch for control of REM sleep. Nature. 2006;441(7093):589-94.

91. Luppi PH, Clement O, Sapin E, Peyron C, Gervasoni D, Leger L, et al. Brainstem mechanisms of paradoxical (REM) sleep generation. Pflugers Arch - Eur J Physiol. 2012;463(1):43-52.

92. Adamantidis AR, Zhang F, Aravanis AM, Deisseroth K, de Lecea L. Neural substrates of awakening probed with optogenetic control of hypocretin neurons. Nature. 2007;450(7168):420-4.

93. Luppi PH, Clement O, Fort P. Paradoxical (REM) sleep genesis by the brainstem is under hypothalamic control. Curr Opin Neurobiol. 2013;23(5):786-92.

94. Hassani OK, Lee MG, Jones BE. Melanin-concentrating hormone neurons discharge in a reciprocal manner to orexin neurons across the sleep-wake cycle. Proc Natl Acad Sci U S A. 2009;106(7): 2418-22.

95. Verret L, Fort P, Gervasoni D, Leger L, Luppi PH. Localization of the neurons active during paradoxical (REM) sleep and projecting to the locus coeruleus noradrenergic neurons in the rat. J Comp Neurol. 2006;495:573-86.

96. Jego S, Glasgow SD, Herrera CG, Ekstrand M, Reed SJ, Boyce R, et al. Optogenetic identification of a rapid eye movement sleep modulatory circuit in the hypothalamus. Nat Neurosci. 2013;16(11):1637-43.

97. Scherfler C, Frauscher B, Schocke M, Iranzo A, Gschliesser V, Seppi K, et al. White and gray matter abnormalities in idiopathic rapid eye movement sleep behavior disorder: a diffusion-tensor imaging and voxel-based morphometry study. Ann Neurol. 2011;69(2):400-7.

98. Provini F, Vetrugno R, Pastorelli F, Lombardi C, Plazzi G, Marliani AF, et al. Status dissociatus after surgery for tegmental ponto-mesencephalic cavernoma: a state-dependent disorder of motor control during sleep. Mov Dis: Off J Mov Dis Soc. 2004;19(6):719-23.

99. Tippmann-Peikert M, Boeve BF, Keegan BM. REM sleep behavior disorder initiated by acute brainstem multiple sclerosis. Neurology. 2006;66(8):1277-9.

100. Garcia-Lorenzo D, Longo-Dos Santos C, Ewenczyk C, LeuSemenescu S, Gallea C, Quattrocchi G, et al. The coeruleus/ subcoeruleus complex in rapid eye movement sleep behaviour disorders in Parkinson's disease. Brain: J Neurol. 2013;136(Pt 7):2120-9.

101. Iranzo A, Tolosa E, Gelpi E, Molinuevo JL, Valldeoriola F, Serradell M, et al. Neurodegenerative disease status and postmortem pathology in idiopathic rapid-eye-movement sleep behaviour disorder: an observational cohort study. Lancet Neurol. 2013;12(5):443-53.

102. Boeve BF, Silber MH, Saper CB, Ferman TJ, Dickson DW, Parisi JE, et al. Pathophysiology of REM sleep behaviour disorder and relevance to neurodegenerative disease. Brain: J Neurol. 2007;130(Pt 11):2770-88. 